\title{
WEATHER FORECASTING VIA THE MODELING OF TEMPERATURE
}

DOI: http://dx.doi.org/10.18509/GBP.2018.15

UDC: $\mathbf{5 5 1 . 5 0}$

\author{
Bhupendra Nath Tiwari \\ S. Chandra Kishore \\ Ninoslav Marina \\ University of Information Science \& Technology "St. Paul the Apostle" - Ohrid, \\ Ohrid, Republic of Macedonia
}

\begin{abstract}
The present work emphasizes on the geographical perspectives of temperature fluctuations and related environmental issues. In this scenario, we study weather forecasting via the techniques of simulated annealing. Simulated annealing provides significant tools in optimizing complex problems, relating socioeconomic importance in the contemporary sciences, engineering, economics and statistics. For the given shape parameter of the probing material and artificial time, we examine optimization properties of the environmental temperature beyond the notion of Boltzmann machine. As per the above analysis, for a given finite artificial temperature, we find that its fluctuations over the system parameters yield a stable configuration, depending on the signature of parameters. In addition, our investigation anticipates computational weather forecasting and modeling of climate changing effects in the light of quenching, optimal theory, artificial intelligence and neural networks.
\end{abstract}

Keywords: Weather forecasting, optimization, simulated annealing, artificial intelligence, neural networks

\section{INTRODUCTION}

Weather has become an important research topic in the field of geographic domain [1]. The impulsive characteristics of the weather that keep changing with various parameters and its socio-economical significance interpolate researchers to develop accurate weather forecasting algorithms. Among various developments occurring in this field of research include the contemporary approaches to simulate complex numerical and physical systems with the aid of optimization models. Such techniques have helped the mankind for an improved prediction of weather in advance with a high degree of reliability.

In general, there are two widely used methods for the weather forecasting such as numerical and statistical methods. Numerical weather forecasting methods involve as dynamic modeling of atmospheric phenomena [2]. However, the statistical methods refer to dynamics interpretations of the atmospheric system based on the already available information. For example, statistical methods mainly work with artificial neural network (ANN) mostly using back propagation techniques [3]. Such techniques have gained much attention in recent decades in the meteorological domain via the time series predictions. In the current scenario, precise and reliable weather predictions are considered to be a challenging task for the researchers. The solution for an accurate weather forecasting can be visualized from the perspective of physical sciences using the interpolation of NavierStokes equation, mass continuity equation, first law of thermodynamics and ideal gas 
equation [4]. These formulations help us to study the changes in the space and time arising from the wind, temperature, pressure and density fluctuations in the atmosphere. With the support of numerical methods, these equations could solve both the resolved and unresolved scales of the motion, resulting in a temperature gradient. To date there are numerous complex models available for the global weather prediction, local area modeling for high impact weather and air-quality predictions. Although, knowledge in physical process, ensemble theory and model initializations are mandatory for resolving the future technology challenges. This involves computing and perspective observations towards an accurate weather forecasting.

Parameterization plays a major role in the prediction of the weather by creating an interface between the atmosphere and earth surface [5]. Parameterization mainly consists of diffusive, radiative and conductive effects in the atmosphere domain. The predictive behavior of parameterization helps us in analyzing various aspects of simulated weather, viz. fluctuations arising from the temperature, wind, clouds and precipitation. Numerical weather forecasting models make use of the similar formulations used in such parameterizations for short-to-medium range forecasting and creating algorithms for model initialization.

While dealing with various problems in this disciple of the physical geography, one generically focuses on the minimization of an appropriate energy or cost function that is defined in a D-dimensional continuous space [6]. There are two different situations to be taken into consideration, i.e., if the energy/ cost is a convex function with a single minimum, the problem could be solved by the gradient descent method. However, one requires more sophisticated methods, e.g., simulated annealing to solve the same, if the energy or cost is a nonconvex function with multiple extrema. This technique has an advantage of introducing more than one artificial temperature where the concerning probing material could be cooled gradually. The artificial temperatures represent the source of stochasity that is well appropriate for detrapping from a local minimum. In classical viewpoint, the mandatory condition for achieving unit probability at the end of the global minimum is to have a logarithmic decrease of the temperature in time. This algorithm is called classical simulated temperature or Boltzmann machine.

In this paper, we focus to solve large-scale optimization problems which are unmanageable by such conventional or existing (combinatorial) methods. Namely, our focus relies on configurations with a large number of objects analogous to the traveling salesman problem [7]. For such problems, it is known [7] that one can hardly find its optimum solution at large scale, thus our proposal is to optimize the undermining profile temperature with respect to its model parameters and find the best suited values. This renders an apt solution for complex optimization problems. An importance of our analysis arises in finding good solutions to aforementioned optimization problems even in the presence of noises in the data. As mentioned above, as far as our optimization analysis is concerned, we design simulated annealing to mimic an optimal process. This is traversed by mislocated atoms in a given metal when it is slowly heated and then slowly cooled in time.

Below, we provide a brief account of numerical weather forecasting, simulated annealing, Metropolis algorithm, fluctuation analysis of the artificial temperature under variations of the model parameters, viz. the shape of the probing material and artificial time, in the next section. In section 3, we discuss results arising from fluctuations of the model parameters. Finally, in section 4, we conclude our paper with directions for future developments. 


\section{BACKGROUND MATERIAL AND DEVELOPMENT OF THE MODEL}

In this section, we recall the notion of the numerical weather forecasting, simulated annealing, Metropolis algorithm and fluctuation theory based optimization analysis of the artificial temperature under fluctuations of its model parameters, viz. the shape/ dimension of the probing material and artificial time of the scheduling algorithm.

\section{What is Numerical Weather Forecasting?}

Numerical weather forecasting (NWF) or the process of numerical weather prediction (NWP) is a mathematical procedure that is implemented via the field of computer science with a prior given code. This is executed mainly as an algorithm with included mathematical equations and formulas for optimally calculating the environmental parameters of the model such as the wind speed, humidity, hot or cold as higher or lower values of the temperature.

Overall, NWF measures these variables, whereby we design it to optimally calculate them and to see how the weather will locally evolve in near future. In fact, with certain calculations the algorithm should predict how the climate will change in future in a given geographic region, whereby we generically wish to forecast "future" weather conditions.

\section{What is Simulated Annealing?}

Simulated annealing was firstly introduced when scientists were heating and cooling a heavy metal object to see how its temperature has increased or decreased on the graph and to mark those results in order to find an appropriate optimization of the temperature. In this concern, there are many simulated annealing based algorithms that can carry out such optimizations, namely to find the global minimum or maximum of an objective function. This supports us in finding good results according to previous calculations, in an algorithm for a given set of formulas and equations. The simulated annealing algorithm makes an estimation between a large number of gathered results to make them more precise and the concerning computations accurate for the perspective weather forecasting.

\section{Metropolis Algorithm}

Simulated annealing offers an enhanced optimization strategy by invoking the role of the Metropolis algorithm [8]. As per this hypothesis, some trades that are not lowering the mileage/ energy are accepted when they allow the optimizer to search more of the existing solution space. Mathematically, given an arbitrary trade/ state, let $\Delta D$ be the change in the corresponding distance with the convention that it takes a negative value for "good" trades, and a positive value for "bad" trades, $T$ be an artificial or synthetic temperature concerning the probing material, and $R(0,1)$ be a random number in the unit interval $[0,1]$. From the above perspective, in the Boltzmann's unit of the temperature [8], Metropolis algorithm filters out the undermining "bad" trades as per the criterion

$$
e^{\Delta D / T}>R(0,1)
$$

Here, $D$ is termed as the cost function, which is physically equivalent to the free energy in the case of the annealing of a heavy metal. To be precise, the temperature parameter $T$ is actually rescaled to $k T$, where $k$ is the standard Boltzmann's constant and $T$ is the surface temperature of the probing material measured in the unit of absolute Kelvin scale. In practice, when $T$ takes a large value, many "bad" trades are accepted by Metropolis optimizer [8], whereby a large portion of the solution space is searched. In 
general, in order to have a better result, objects under the analysis of simulated annealing are chosen randomly. However, there exist other refinements of the above mentioned Metropolis analysis, whose parametric optimization is left open for further investigations. As mentioned above, our optimization procedure follows an analogy of the cooling of a metal. Therefore, by making several trades and noticing that the concerned cost function slowly reduces, we may lower the temperature to a smaller value. This reduces the size of "bad" trades in the search space. By repeating this process several times, i.e., when the temperature gets sufficiently reduced, we quench the process by allowing only good trades as the possible solutions. This yields a local minimum of the cost function. Indeed, there exists a number of advanced "annealing schedules" to lower the temperature, but the results are mostly insensitive to such refinements and minor details.

For example, the threshold acceptance strategy [9] accepts all the good trades, as any of the bad trades raising the undermining cost function above its chosen threshold value. Hereby, we may periodically lower the threshold as the annealing temperature. This provides an alternative to the Boltzmann criterion. Such simulations can be time effective, as we need not to either perform the exponentiation or generate the random number as in Eqn.(2.1).

\section{Stability Analysis via Fluctuation Theory}

In the sequel, for given shape parameter $q$ and artificial time $t$, we wish to determine the optimum value of the temperature $T(q, t)$ via its symmetric Hessian matrix $H=$ $\left(T_{i j}\right)_{2 \times 2}$. The entries of $H$ are defined as the second order partial derivatives of $T(q, t)$ as $\left(T_{i j}\right)_{2 \times 2}=\partial_{i} \partial_{j} T(q, t)$, where $i, j \in\{q, t\}$.

To find an optimal value of the above artificial temperature $T(q, t)$, we require vanishing of the undermining flow components $\left\{T_{q}, T_{t}\right\}$, that is to find roots of the equalities

$$
T_{q}=0, T_{t}=0
$$

The concerning (in)stability conditions are determined by the determinant of $H$ defined as

$$
D:=T_{q q} T_{t t}-T_{q t}^{2}
$$

In this concern, it is worth emphasizing that the sign of $D$ plays an important role. Namely, there exists a local minimum or stable configuration if $D>0$ with the shape capacity $T_{q q}>0$ or the artificial time capacity $T_{t t}>0$. Similarly, there is a local maximum of $T(q, t)$ or the undermining fluctuating configuration becomes unstable if $D>0$ with $T_{q q}<0$ or $T_{t t}<0$. On the other hand, it is well known that the system goes under a saddle behavior when the Hessian determinant $D$ of the temperature profile has a negative value, viz. $D<0$. For the case of the vanishing discriminant $D(q, t)=0$, the stability test of the temperature $T(q, t)$ gives a degeneration curve, as displayed in Eqn.(2.24) in the next section.

\section{RESULTS AND DISCUSSION}

Under fluctuations of the above configuration parameters $\{q, t\}$, it is known [6] that the artificial temperature $T(q, t)$ is defined as: 


$$
\mathrm{T}(\mathrm{q}, \mathrm{t})=T(q) \frac{2^{q-1}-1}{(1+t)^{q-1}-1}
$$

In order to simplify the subsequent expressions, the denominator of $T(q, t)$ is defined as

$$
d:=(1+t)^{q-1}-1
$$

A direct calculation shows that we have the following shape flow component

$$
T_{q}(q, t)=\frac{1}{d^{2}}\left[\left\{2^{q-1} d \ln 2-\left(2^{q-1}-1\right)(1+t)^{q-1} \ln (1+t)\right\} T(q)+\left(2^{q-1}-1\right) d \frac{d T}{d q}\right]
$$

Similarly, it follows that the flow component corresponding to the artificial time $t$ is given by

$$
T_{t}(q, t)=-(q-1) T(q) \frac{(1+t)^{q-2}}{d^{2}}\left(2^{q-1}-1\right)
$$

Now from the Eqns.(3.3, 3.4), we see that the flow components $\left\{T_{q}, T_{t}\right\}$ vanish as per the following cases. The flow component $T_{t}$ as in Eqn.(3.4) vanishes whenever we have $q=$ 1 or $t=-1$. In both the above cases, we observe that the flow component $T_{q}$ as in Eqn.(3.3) vanishes according as a first order differential equation.

As mentioned in the foregoing section, under fluctuations of the model parameters $t$ and $q$, a direct differentiation of the artificial time flow component $T_{t}$ as in Eqn.(3.4) with respect to the artificial time $t$ yields the following artificial time capacity

$$
T_{t t}(q, t)=-\frac{n_{1} n_{2}}{d^{3}}
$$

where the above factors $\left\{n_{1}, n_{2}\right\}$ are given by the expressions

$$
\begin{gathered}
n_{1}=T(q)\left(2^{q-1}-1\right)(q-1)(1+t)^{q-1} \\
n_{2}=(q-2)(1+t)^{q-1}-2(q-1)(1+t)^{q-3}-(q-2)
\end{gathered}
$$

Similarly, by differentiating the flow component $T_{q}$ as in Eqn.(3.3) with respect to the shape/ dimension of the probing material $q$, we find that its capacity reads as per the following

$$
T_{q q}(q, t)=\frac{h_{1}}{d}-\frac{h_{2}}{d^{2}}+2 \frac{h_{3}}{d^{3}},
$$

where the components $\left\{h_{1}, h_{2}, h_{3}\right\}$ as in the above Eqn.(3.8) are given as

$$
\begin{gathered}
h_{1}=\left(2^{q-1}-1\right) \frac{d^{2} T}{d q^{2}}+2^{q} \ln 2 \frac{d T}{d q}+2^{q-1}\left(2^{q-1}+1\right)(\ln 2)^{2} T(q) \\
h_{2}=(1+t)^{q-1} \ln (1+t)\left\{2\left(2^{q-1}-1\right) \frac{d T}{d q}+\left(2^{q} \ln 2+\left(2^{q-1}-1\right) \ln (1+t)\right) T(q)\right\}
\end{gathered}
$$




$$
h_{3}=\left(2^{q-1}-1\right)(1+t)^{2 q-2}\{\ln (1+t)\}^{2} T(q)
$$

On the other hand, the correlation between the shape/ dimension of the probing material $q$ and artificial time $t$ is given by the mixed partial derivative $T_{q t}(q, t)$ of the profile temperature $T(q, t)$. Physically, in the $q t$-plane, fluctuations with respect to both the model parameters $q$ and $t$ may dependent on each other. In general, their dependency is defined as per the mixed second partial derivative of $T(q, t)$ as

$$
T_{q t}(q, t)=\frac{\partial^{2}}{\partial q \partial t} T(q, t)
$$

By differentiating the flow component $T_{t}$ as in Eqn.(3.4) with respect to the shape of the probing material $q$, it is not difficult to show that we have the following correlation

$$
T_{q t}(q, t)=\frac{c_{1}}{d^{2}}-2 \frac{c_{2}}{d^{3}}
$$

where the components $\left\{c_{1}, c_{2}\right\}$ as given in Eqn.(3.13) reads as per the expressions

$c_{1}=(q-1)(1+t)^{q-2}\left\{\left(2^{q-1}-1\right) \frac{d T}{d q}+\left(\left(2^{q-1}-1\right) \ln (1+t)+2^{q-1} \ln 2\right) T(q)\right\}$

$$
c_{2}=(q-1)(1+t)^{2 q-3}\left(2^{q-1}-1\right) \ln (1+t) T(q)
$$

Herewith, we see that there is no correlation in the system if $\left\{c_{1}, c_{2}\right\}$ satisfy $2 c_{2}=c_{1} d$. In the sequel, in order to examine the global stability properties of the temperature fluctuations in terms of the model parameters $\{q, t\}$, we need to compute the discriminant

$$
D(q, t):=T_{q q}(q, t) \cdot T_{t t}(q, t)-T_{q t}(q, t)^{2}
$$

By substituting the values of the heat capacities $\left\{T_{q q}(q, t), T_{t t}(q, t)\right\}$ from Eqns. $(3.5,3.8)$ and the undermining correlation component $T_{q t}(q, t)$ as depicted in Eqn.(3.13), we arrive at the following discriminant

$$
D(q, t)=\frac{1}{d^{6}}\left\{n_{1} n_{2}\left(h_{2} d-h_{1} d^{2}-2 h_{3}\right)+4 c_{1} c_{2}-c_{1}{ }^{2}-4{c_{2}}^{2}\right\},
$$

where the capacity components $\left\{n_{1}, n_{2}, h_{1}, h_{2}, h_{3}\right\}$ are given in Eqns.(3.6, 3.7, 3.9-3.11) and the corresponding correlation components $\left\{c_{1}, c_{2}\right\}$ read as in Eqns.(3.14, 3.15).

Herewith, the temperature profile $T(q, t)$ has a saddle point behavior, whenever we have negative signature of the above discriminant, viz. $D(q, t)<0$. In other words, the Eqn.(3.17) shows that the system becomes saddle when $\left\{n_{1}, n_{2}, h_{1}, h_{2}, h_{3}, c_{1}, c_{2}\right\}$ satisfy the inequality

$$
n_{1} n_{2}\left(h_{2} d-h_{1} d^{2}-2 h_{3}\right)<c_{1}{ }^{2}+4 c_{2}{ }^{2}-4 c_{1} c_{2}
$$


The system remains stable or unstable according as the temperature $T(q, t)$ has a minimum or maximum about its fixed points. First of all, this happens if we have $D(q, t)>0$. That is, in order to find a stable configuration or a minimum value of $T(q, t)$ as mentioned in section 2.4 , we must have

$$
n_{1} n_{2}\left(h_{2} d-h_{1} d^{2}-2 h_{3}\right)>c_{1}^{2}+4 c_{2}^{2}-4 c_{1} c_{2}
$$

with either of the following constraints

$$
\left\{\begin{array}{c}
n_{1} \text { and } n_{2} \text { have the opposite sign for } d>0 \\
n_{1} \text { and } n_{2} \text { have the same sign for } d<0
\end{array}\right.
$$

$$
\left\{\begin{array}{l}
h_{2} d-h_{1} d^{2}-2 h_{3}<0 \text { for } d>0 \\
h_{2} d-h_{1} d^{2}-2 h_{3}>0 \text { for } d<0
\end{array}\right.
$$

Notice that we have an unstable configuration, that is, the temperature $T(q, t)$ attains a maximum if it satisfies Eqn.(3.19) with the opposite conditions of Eqns.(3.20, 3.21). Namely, as outlined in section 2.4, we have a maximum of $T(q, t)$ if Eqn.(3.19) holds with either of the following conditions

$$
\begin{aligned}
\text { (i) } \begin{array}{l}
n_{1} \text { and } n_{2} \text { have the same sign for } d>0 \\
n_{1} \text { and } n_{2} \text { have the opposite sign for } d<0
\end{array} \\
(3.22) \\
\text { (ii) }\left\{\begin{array}{l}
h_{2} d-h_{1} d^{2}-2 h_{3}>0 \text { for } d>0 \\
h_{2} d-h_{1} d^{2}-2 h_{3}<0 \text { for } d<0
\end{array}\right. \\
(3.23)
\end{aligned}
$$

The stability test of $T(q, t)$ fails when it has a vanishing discriminant, viz. we have $D(q, t)=0$. Alternatively, from Eqn.(3.19), we see that this happens when the stability and correlation components $\left\{n_{1}, n_{2}, h_{1}, h_{2}, h_{3}, c_{1}, c_{2}\right\}$ satisfy the equality

$$
n_{1} n_{2}=\frac{c_{1}{ }^{2}+4 c_{2}{ }^{2}-4 c_{1} c_{2}}{h_{2} d-h_{1} d^{2}-2 h_{3}}
$$

In this case, we say that the temperature function $T(q, t)$ is too flat. Namely, we need a higher derivative test to examine stability properties of the profile temperature $T(q, t)$ under fluctuations of the system parameters $\{q, t\}$. Indeed, there exists certain analysis based on a jerk and other parameters which are defined as the third or higher order derivatives of the function $T(q, t)$. In the light of the simulated annealing based optimizations towards an apt probe designing, we leave such investigations open for a future research.

\section{CONCLUSION}

The idea of this paper is to explain the notion of Numerical Weather Forecasting in general and to give its interpretation in the light of optimization theory. Here, we have 
explained the simulated annealing process, and its optimization how the scientists could implement it in forecasting of weather and its meanings in connection with the artificial intelligence.

In the sequel, we have explored mathematical perspectives for a given artificial temperature profile and find its flow components, capacities under different values of the shape parameter $q$ of the probing material and the artificial scheduling time $t$. We have obtained the flow components as the first order derivatives of the artificial temperature with respect to $q$ and $t$ values, and the fluctuation capacities as that of the pure second order derivatives. The system stability criterion is determined by the determinant of the corresponding fluctuation matrix $H$ according to the second order of variations of the artificial temperature $T(q, t)$ with respect to $q$ and $t$. Also, we find its mixed derivative with respect to $\{q, t\}$ together as the formula as in Eqn.(3.13). This explicitly gives the undermining system correlation.

In nutshell, we have implemented fluctuation theory analysis from the above gathered values of the system capacities and correlation. Subsequently, by storing them in the fluctuation matrix, we have offered optimal properties of the artificial temperature under different parameter values. The corresponding initial value problem, its generation as a first order differential equation and computational optimizations [10] are left open for future research. In the light of temperature variations in a chosen geographic region, our model finds utilities for accurate prediction of weather with variation of time and probing material dimension.

\section{REFERENCES}

[1] Kendon, Elizabeth J., et al. "Heavier summer downpours with climate change revealed by weather forecast resolution model." Nature Climate Change 4.7 (2014): 570.

[2] Baldauf, Michael, et al. "Operational convective-scale numerical weather prediction with the COSMO model: description and sensitivities." Monthly Weather Review 139.12 (2011): 38873905.

[3] Glahn, Harry R. "Statistical weather forecasting." Probability, statistics, and decision making in the atmospheric sciences (1985): 289-335.

[4] Bauer, Peter, Alan Thorpe, and Gilbert Brunet. "The quiet revolution of numerical weather prediction." Nature 525.7567 (2015): 47.

[5] Grell, Georg A., and Saulo R. Freitas. "A scale and aerosol aware stochastic convective parameterization for weather and air quality modeling." Atmospheric Chemistry \& Physics Discussions 13.9 (2013).

[6] Tsallis, Constantino, and Daniel A. Stariolo. "Generalized simulated annealing." Physica A: Statistical Mechanics and its Applications 233.1-2 (1996): 395-406.

[7] Skiena, S. S. "Traveling Salesman Problem." §8.5.4 in The Algorithm Design Manual. New York: Springer-Verlag, pp. 319-322, 1997.

[8] Metropolis, N.; Rosenbluth, A. W.; Rosenbluth, M.; Teller, A. H.; and Teller, E. "Equation of State Calculations by Fast Computing Machines." J. Chem. Phys. 21, 1087-1092, 1953.

[9] Dueck, G. and Scheuer, T. "Threshold Accepting: A General Purpose Optimization Algorithm Appearing Superior to Simulated Annealing." J. Comp. Phys. 90, 161-175, 1990.

[10] Lynch P., "The origins of computer weather prediction and climate modeling", ELSEVIER - Journal of Computational Physics 227, 3431-3444, 2008 